\title{
Preface to the Special Issue Dedicated to Professor Alexander Davidovich loffe
}

\section{Huynh Van Ngai ${ }^{1} \cdot$ Hoang Xuan Phu ${ }^{2} \cdot$ R. Tyrrell Rockafellar ${ }^{3} \cdot$ Michel Théra $^{4,5}$ (1)}

Published online: 25 July 2019

(c) Vietnam Academy of Science and Technology (VAST) and Springer Nature Singapore Pte Ltd. 2019

It is a great honor and privilege to dedicate this issue of "Vietnam Journal of Mathematics" to Professor Alexander Davidovich Ioffe, a true friend and one of the worldwide most important experts of Variational Analysis, Optimal Control and Optimization.

This special issue contains fifteen research papers contributed by highly distinguished mathematicians, colleagues and friends of Professor Alexander Davidovich Ioffe. These contributions cover topics in both theoretical and applied aspects and show the state of the art in many directions of the areas in which Alex has made major contributions. Some of the authors have collaborated with Alex at some time of his career and all of them want to show their appreciation and respect for Alex's work developed along the years. Let us mention that a detailed presentation of the life and the mathematical contributions of Alex is given by the editors in the first article of this special issue.

Michel Théra

michel.thera@unilim.fr

Huynh Van Ngai

ngaivn@yahoo.com

Hoang Xuan Phu

hxphu@math.ac.vn

R. Tyrrell Rockafellar

rtr@uw.edu

1 Department of Mathematics, Quy Nhon University, Quy Nhon, Binh Dinh Vietnam

2 Institute of Mathematics, Vietnam Academy of Science and Technology, Hanoi, Vietnam

3 Department of Mathematics, University of Washington, Seattle, WA 98195-4350, USA

4 Université de Limoges, Limoges, France

5 Centre for Informatics and Applied Optimisation, Federation University Australia,

Ballarat, Australia 
All submissions passed through the usual for Vietnam Journal of Mathematics rigorous reviewing process. In order of submission date, the contributions are as follows.

Variational convexity and the local monotonicity of subgradient mappings, by R. Tyrrell Rockafellar;

A contribution to Reich's conjecture, by Dominique Azé and Jean-Noël Corvellec; On the existence of Lipschitz continuous optimal feedback control, by Asen L. Dontchev, Mikhail I. Krastanov, and Vladimir M. Veliov;

A unified characterization of nonlinear scalarizing functionals in optimization, by Gemayqzel Bouza, Ernest Quintana, and Christiane Tammer;

Convexity and closure in optimal allocations determined by decomposable measures, by Zvi Artstein;

On Nash-Moser-Ekeland inverse mapping theorem, by Radek Cibulka and Marian Fabian;

A versatile approach to metric regularity, openness and Lipschitzian properties, by Jean-Paul Penot;

Perturbations of supinf problems with constraints, by Damien Gaumont, Detelina Kamburova, and Julian P. Revalski;

Nonsmooth Kantorovich-Newton methods: Hypotheses and auxiliary problems, by Diethard Klatte and Bernd Kummer;

Problems in the calculus of variations on unbounded intervals - FourierLaguerre analysis and approximations, by Sabine Pickenhain and Angie Burtchen;

Local linear convergence for inexact alternating projections on nonconvex sets, by Dmitriy Drusvyatskiy and Adrian S. Lewis;

Free end-time optimal control problems: Conditions for the absence of an infimum gap, by Richard B. Vinter;

Metric regularity relative to a cone, by Huynh Van Ngai, Nguyen Huu Tron, and Michel Théra;

Subdifferential stability and subdifferential sum rules, by Marc Lassonde;

Alexander Davidovich Ioffe: A Life of Contributions, by Huynh Van Ngai, Hoang Xuan Phu, R. Tyrrell Rockafellar and Michel Théra.

We would like to thank Vietnam Journal of Mathematics, as well as all the authors and all the many referees for giving us the opportunity to honor Alex through this special issue.

In honoring Alex on the occasion of his 80th birthday, we wish him great health and many fruitful happy years, full of exciting research!

April 2019

Publisher's Note Springer Nature remains neutral with regard to jurisdictional claims in published maps and institutional affiliations. 